\title{
Influence of Gingival Display on Smile Esthetics
}

Pavan Kumar Mamillapalli ${ }^{1}$ Vasu Murthy Sesham², Praveen Kumar Neela ${ }^{3}$, Shiva Prasad Mandaloju ${ }^{4}$, Sreekanth Keesara ${ }^{5}$, Abdul Rehman Ahmed Khan ${ }^{6}$

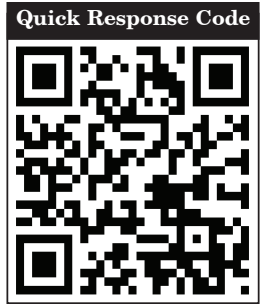

doi: $10.5866 / 2016.8 .10232$

${ }^{1}$ Professor

${ }^{2}$ Professor \& HOD

${ }^{3}$ Reader

${ }^{4 \& 5}$ Senior Lecturer

${ }^{6}$ Former PG Student

Department of Orthodontics,

Kamineni Inst of Dental Sciences,

Narketpally.

Article Info:

Received: October 12, 2016

Review Completed: November 11, 2016

Accepted: December 13, 2016

Available Online: December, 2016 (www.nacd.in)

(C) NAD, 2016 - All rights reserved

Email for correspondence:

pavan.dr@gmail.com

\begin{abstract}
:
The objective of the present study is to assess which level of gingival exposure is considered more esthetic and to compare the differences in opinion among orthodontists, dentists and laypersons in relation to the gingival exposure for the smile esthetics. One photograph presenting well aligned teeth and a pleasing smile was selected for the study. By considering the patient's original photographs as ideal, manipulations were done with the Photoshop software by decreasing $4 \mathrm{~mm}$ of gingival display, $2 \mathrm{~mm}$ of gingival display and also increasing $4 \mathrm{~mm}$ and $2 \mathrm{~mm}$ of gingival display. Chi square test was performed to find out whether any statistical significance among the three groups (Dentists, Orthodontists, Lay persons) was present. The results show that $2 \mathrm{~mm}$ decrease of gingival display was considered as more aesthetic by all the three groups.
\end{abstract}

Key words: Smile esthetics, Gingival display, Esthetic harmony.

\section{INTRODUCTION}

Facial attractiveness has been suggested to have an influence on personality development and social interaction (Adams, 1977; Feingold, 1992; Thompson et al., 2004). The smile plays an important role in facial expression. Facial attractiveness and smile aesthetics are strongly related to each other. Individuals mainly focus on another person's eyes and mouth during interpersonal interaction (Miller, 1970), and the smile ranks second only to the eyes as the most important feature in facial attractiveness (Goldstein,
1969). Therefore, an attractive, well-balanced smile is a highly regarded treatment objective, along with creating a functional occlusion. An attractive smile depends not only on components such as tooth size, shape, colour, and position but also on the amount of visible gingivae and the framing of the lips (Van der Geld et al., 2007). ${ }^{1}$

A 'gummy' smile results from a combination of factors such as vertical maxillary excess, increased overjet, increased overbite, a short upper lip, and a short incisor crown length (Allen, 1988). ${ }^{1}$ 
Although many orthodontists and clinicians consider gingival smiles as non-desired, this characteristic might not necessarily be unaesthetic to the public eyes. ${ }^{2}$

\section{Materials and Methods}

After taking the informed consent, one photograph presenting well aligned teeth and a pleasing smile was selected for the study

\section{Methodology}

By considering the patient's original photographs as ideal, manipulations were done with the Photoshop software in the following manner:

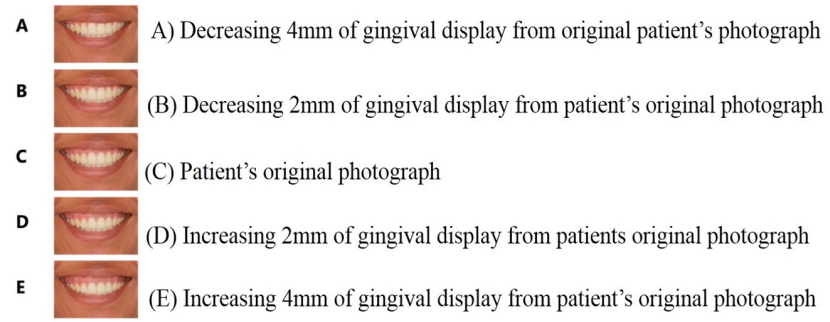

The photographs were shown to various evaluators comprising of 150 people (50 orthodontists, 50 dentists and 50 lay persons) and their opinion was taken through a questionnaire asking "Which smile would you consider to be the best"? Give your opinion.

The opinions of all the evaluators were entered on a Microsoft Excel and sent for statistical analysis

\section{OPINION}

Esthetics

Influence of Gingival Display on Smile

Name:

Designation:

Phone number:

Which smile would you consider to be the best? Please write the respective al phabet: [ ]

\section{RESULTS}

All the statistical analyses were performed with a commercial software package (SPSS 20.0 software). Chi square test was performed to find out the presence of statistical significance among the three groups (Dentists, Orthodontists, Lay persons) (Table 1-4).

\section{DISCUSSION}

Esthetics denotes concern about beauty or appreciation of beauty. "Beauty truly lies in the eye of the beholder", and what is desirable to one might not be so to another. ${ }^{3}$ The perception of esthetics varies from person to person and is influenced by personal experiences and social environments. ${ }^{6} \mathrm{~F}$ or the samereasons, there can be differences of opinion regarding beauty between laypersons and professionals. Diamond reported that what a person finds attractive has much to do with the qualities of the surrounding population; other environmental factors, such as education, are also involved. ${ }^{6}$ Smile esthetics have become a central concern for patients and Orthodontists because this is the primary reason that patients seek Orthodontic treatment and Orthodontists are now using this as a focus for treatment planning. ${ }^{5}$

From a psychological point of view, we can distinguish two dimensions in self-perception of the smile. The ûrst dimension is perception of attractiveness of the smile. This perception is especially dened by the opinions of others and cultural norms. The social dimension (eld of social psychology) is relative to the history of culture. As cultures are changing, perception of attractiveness is changing too. The second dimension in selfperception of the smile is satisfaction with its appearance. This perception originates from the internal view, the inner experience of the individual itself: the individual dimension. ${ }^{4}$

Several studies about esthetic perceptions found that dentists, particularly orthodontists, are less tolerant than the general public for some dental conditions. ${ }^{7}$ Several authors evaluated the esthetic perception of various malocclusions, but little information is available about the perception of the effect of dental asymmetries on smile esthetics. So, the purpose of this study was to evaluate the 
Table 1: Comparison of the ratings given by Dentists, Orthodontists and lay persons $(p=0.001)$.

\begin{tabular}{llllll} 
& A & B & C & D & E \\
Dentist & 23 & 23 & 3 & 0 & 1 \\
\hline Orthodontist & 10 & 39 & 0 & 0 & 0 \\
\hline Lay person & 13 & 12 & 12 & 8 & 5 \\
\hline
\end{tabular}

Chi square test was also performed to find out the presence of statistical significance among the two groups (Dentists and Orthodontists).

Table 2: Comparison of the ratings given by Dentists and Orthodontists $(p=0.004)$.

\begin{tabular}{llllll} 
& A & B & C & D & E \\
Dentist & 23 & 23 & 3 & 0 & 1 \\
\hline Orthodontist & 10 & 39 & 0 & 0 & 0 \\
\hline
\end{tabular}

Chi square test was also performed to find out the presence of statistical significance among the two groups (Dentists and Lay persons).

Table 3: Comparison of the ratings given by Dentists and Lay persons $(p=0.0001)$.

\begin{tabular}{llllll} 
& A & B & C & D & E \\
Dentist & 23 & 23 & 3 & 0 & 1 \\
\hline Lay person & 13 & 12 & 12 & 8 & 5 \\
\hline
\end{tabular}

Chi square test was also performed to find out the presence of statistical significance among the two groups (Orthodontists and Lay persons).

Table 4: Comparison of the ratings given by Orthodontists and Lay persons $(p=0.001)$.

\begin{tabular}{llllll} 
& A & B & C & D & E \\
Orthodontist & 10 & 39 & 0 & 0 & 0 \\
\hline Lay person & 13 & 12 & 12 & 8 & 5 \\
\hline
\end{tabular}

influence of gingival display on smile esthetics by Orthodontists, Dentists and laypersons.

One photograph presenting well aligned teeth and a pleasing smile was selected for the study. By considering the patients original photograph as ideal, manipulations were done with the Photoshop software in the following manner: (A) Decreasing $4 \mathrm{~mm}$ of gingival display from original patient's photograph, (B) Decreasing $2 \mathrm{~mm}$ of gingival display from patient's original photograph, (C) Patient's original photograph, (D) I ncreasing $2 \mathrm{~mm}$ of gingival display from patients original photograph, (E) Increasing $4 \mathrm{~mm}$ of gingival display from patients original photograph. The photographs were shown to various evaluators comprising of 150 people (50 orthodontists, 50 dentists and 50 lay persons) and their opinion was taken through a questionnaire asking "Which smile would you consider to be the best"? Give your opinion.

The results of the present study shows that of 50 orthodontists, 50 dentists and 50 laypersons, [23 dentists have consi dered photograph A as ideal smile photograph, where as the other 23 dentists have considered photograph B as ideal, photograph C was supported by 3 dentists and $\mathrm{E}$ by only 1 dentist]. 
When the ratings given by orthodontists were taken into consideration it shows that [10 orthodontists have considered photograph A as the ideal smile photograph, whereas remaining 40 orthodontists have considered photograph B as ideal]. Among the lay persons category [13 lay persons have considered photograph A as ideal,12 have considered photograph B as ideal, other 12 have considered photograph $C$ as ideal, photograph $D$ is considered as ideal by 8 lay persons and $E$ by remaining 5 persons].

When chi square test was applied to these ratings it showed the presence of statistical significance with ( $p=0.001$ ) between the three groups that is Dentists-Orthodontists-Lay persons. The results of the present study also shows that the maximum ratings were given to Photograph $B$ which had the gingival display in the range of 2 to $3 \mathrm{~mm}$, which shows $2 \mathrm{~mm}$ of decreased gingival display from the original patients smile photograph that is photograph $C$ which shows nearly 3 to $5 \mathrm{~mm}$ of gingival display.

\section{CONCLUSION}

The result of the present study concludes that the photograph B with 2 to $4 \mathrm{~mm}$ of gingival display is considered as the ideal smile photograph according to the maximum Orthodontists, Dentists and Lay persons.

\section{References}

1. Tjan AHL, Dent Dr, Millar CD, The J CP: Some esthetic factors of smile. J Prosthet Dent 1984; 51:24-8.

2. Bruna DC, Bittencourt MAV, and Machado AW. Influence of maxillary canine gingival margin asymmetries on the perception of smile esthetics among orthodontists and laypersons. Am J Orthod Dentofacial Orthop 2014; 145:5563.

3. Roden J ohnson D, Gallerano R, English J. The effects of buccal corridor spaces and arch form on smile esthetics. Am J Orthod Dentofacial Orthop 2005; 127:343-350.

4. Van der Geld P, Oosterveld P, Heck GV, Kuijpers-J agtman AM. Smile attractiveness: self-perception and influence on personality. Angle Orthod 2007; 77:759-75.

5. Charles M. Hulsey. An esthetic evaluation of lip-teeth relationships present in the smile-AmJ Orthod Dentofacial Orthop 1970; 57(2):132-44.

6. C Flores Mir, E Silva, MI Barriga, MO Lagravere and PW Major Lay. Person's perception of smileaesthetics in dental and facial views. J Orthodon 2004; 31:204-9.

7. Beyer J W, Lindauer SJ. Evaluation of dental midline position. Semin Orthod 1998; 4:146-52.

\section{Gain quick access to our journal online View our journal at www.nacd.in}

